Scientific Research and Reviews
(ISSN:2638-3500)

\title{
Ship navigation safety risk assessment based on genetic algorithm and BP neural network
}

\author{
He Zhang ${ }^{1^{*}}$, Zhuyun Shao', Ji Zeng ${ }^{1}$ \\ ${ }^{1}$ Shanghai Maritime University, Shanghai 201306, China.
}

\begin{abstract}
In order to ensure the safe navigation of ships and reduce the occurrence of marine accidents, through the analysis of ship He Zhang literature research, taking into account various aspects such as marine meteorology, cargo loading, ship status and crew quality. Construct a ship navigation safety evaluation body. The BP neural network algorithm is used to design the ship navigation safety risk network structure. The sea damage data is used as the network input sample to train the BP neural network and data fitting. At the same time, the genetic algorithm is introduced to find the individual corresponding to the optimal fitness, and the weight and threshold of the network are further optimized. The purpose is to improve the accuracy of data fitting. The optimized BP neural network evaluation results show that there are many indicators affecting the safety of the ship's navigation, and the relationship between the indicators is complicated. The optimized BP neural network utilizes the characteristics of online adaptive learning, which eliminates the construction of complex

relationships among various indicators within the structure, and solves the difficult problems in ship risk assessment to a certain extent.
\end{abstract} historical navigation safety accident data and related accident Shanghai Maritime University, Shanghai 201306, China.

Keywords: Ship navigation safety; BP neural network; Genetic algorithm; Risk assessment

How to cite this article:

He Zhang, Zhuyun Shao, Ji Zeng. Ship navigation safety risk assessment based on genetic algorithm and BP neural network. Scientific Research and Reviews, 2020; 13:117

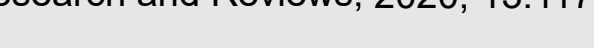




\section{Introduction}

The shipping industry is one of the largest industries in the world. It is one of the industries with the highest risks due to the harsh operating environment, complicated ship technology and large management mobility. At present, many scholars have devoted themselves to the research and analysis of safety management and risk assessment of the marine transportation industry. Yi-yang Xie ${ }^{[1]}$ et al. Studied the accident risk classification of cargo ships and passenger ships in the Yellow and Bohai Seas, and studied ship navigation as a complex system consisting of people, ships, and the environment. Li Yang et al. Studied the accident risk classification of cargo ships and passenger ships in the Yellow and Bohai Seas, and studied ship navigation as a complex system consisting of people, ships, and the environment. Li Yang et al. Studied the accident risk classification of cargo ships and passenger ships in the Yellow and Bohai Seas, and studied ship navigation as a complex system consisting of people, ships, and the environment. Meng-xuan Wang ${ }^{[2]}$ et al. Combined the two methods of ReliefF and ANFIS to construct ship evaluation indicators and risk prediction models, and provided new methods for research in the field of ship safety assessment. Ai-yuan Duan [3] et al. Established an unascertained measurement model to carry out a comprehensive evaluation of the risk factors, the possibility of accidents, and the degree of loss and injury of the ship system. However, the above method is susceptible to subjective factors, and little consideration is given to the correlation characteristics of factors affecting ship safety, which cannot reflect the characteristics of continuous changes between parameters, and is not rigorous in the evaluation process and conclusions.

Neural networks ${ }^{[4]}$ have a wide range of efficient self-learning capabilities, high-speed optimization capabilities, and associative storage capa- bilities. Among them, BP neural networks have a strong nonlinear processing capability as a forward feedback network, and can map out complex nonlinear relationships between sequences. At present, in the field of ship safety research, it is mostly applied to the evaluation of a certain aspect of a ship or a specific system. Jing-kun Xiao ${ }^{[5]}$ et al. Used a BP neural network to establish a screening and evaluation model that can reflect the level of oil spill risk in the research area. Jin-hao Wang ${ }^{[6]}$ et al. Selected 400 fishing boat accident cases and divided them into multiple levels to use BP neural network algorithm to provide early warning of the risk level of fishing boat navigation safety, and the results are basically consistent with the actual accident status of fishing boats. Although the BP neural network has good learning and fault tolerance for data processing, in practice, gradient descent learning methods are often used, and it is easy to fall into the local minimum, causing oscillations and slower convergence ${ }^{[7]}$. The traditional BP neural network is optimized to obtain better initial network weights and thresholds, making the assessment of ship navigation safety more accurate.

\section{Influencing factors of ship navigation sa- fety}

The research of ship navigation safety mainly uses theories and methods of safety system engineering to identify, predict, eliminate or control the unsafe factors and various accidents that may occur in the course of ship navigation, thereby making certain losses, efficiency and other factors the number of accidents is reduced under the constraints of the vehicle, and the best safe navigation conditions of the ship under these conditions are sought. The construction of ship navigation safety system in this paper mainly includes four factors: marine environment factor, cargo loading factor, ship factor and man-

agement factor. 


\subsection{Marine environmental factors}

During the voyage, the ship is always associated with wind and waves, accompanied by tidal waves, and is greatly affected by external natural conditions such as climate and sea conditions. The environmental conditions of ship navigation mainly include the conditions of meteorological sea conditions, the geographical environment of the sea area, the traffic environment of the sea area, and the information environment of the sea area ${ }^{[8]}$. This article mainly starts from the meteorological sea conditions and studies the influence of sea wind (cross wind) and waves on navigation safety. Under the influence of cross wind, the ship will roll and sway, which will cause the ship to capsize when it is serious, which will directly affect the safety of the ship. The waves cause great resistance to the ship in sailing, not only will the sailing speed be lost, but also a series of heave and sway motions. In the case of strong wind and waves, it will cause tilt, deck waves, hull structure damage, etc Navigational safety is one of the most direct and influential factors.

\subsection{Ship factors}

The condition of the ship is of great significance to the study of the evaluation system. The ship factor is mainly considered from the ship itself and the ship management ${ }^{[9]}$. Based on the scientific and operational considerations of safety risk assessment, the ship factor index system is constructed from four aspects: ship structure, ship equipment, ship basic information, and ship management information (as shown in Figure 1). Levels: The first level includes four primary indicators: ship structure, ship equipment, ship basic information and ship management information; the second level includes hull strength, structural integrity, anti-sinking, navigation and signal equipment, and ship power 13 secondary indicators including equipment, safety and emergency equipment, machinery and equipment, ship tonnage, ship type, class, age, historical accidents, management company, etc.

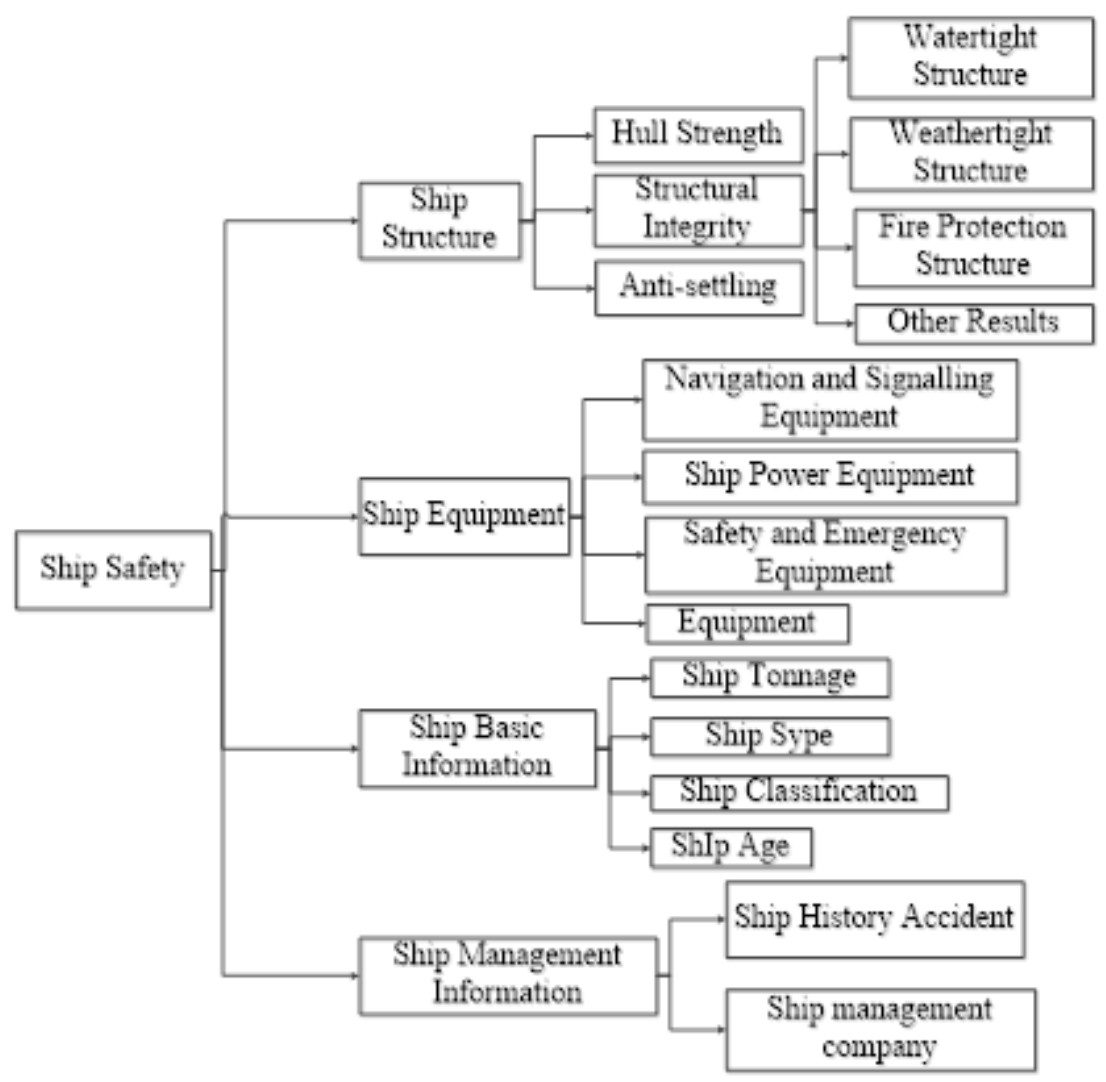

Fig 1 Safety evaluation index of ship factors 


\subsection{Cargo loading factors}

The cargoes carried by ships in the transportation process are different, including liquid cargo (crude oil, wildflower nature, chemicals, etc.), dry bulk cargo (ore, coal, gravel, etc.), general cargo (logs, rolled steel, etc.), containers, etc. In the safety of navigation, not only the value of the goods should be considered, but also the attributes of the cargo, reasonable stowage, and tethering, etc. must be comprehensively considered.

\subsection{Human factors}

With the continuous innovation of modern technology, the shipbuilding industry is developing in the direction of new type, automation, and technology, and the functions of marine equipment and systems are comprehensive and refined. This puts forward relatively high requirements for marine personnel in terms of basic knowledge, operation control, and maintenance. $80 \%$ of the causes of maritime accidents recognized by the international maritime community are caused by human factors ${ }^{[10]}$. Therefore, it is inevitable to consider the influence of human factors in the navigation safety risk assessment. The literature [11] focuses on historical conditions such as historical error records, historical voyage captain evaluations, and other working conditions such as post and experience value of the same post.
Factor system. In addition, it includes the physical and psychological conditions of the crew and the ability to update knowledge.

\subsection{Management factors}

The role of management factors in modern shipping security is becoming increasingly important. A large number of accidents involved ship management and crew management, and the ship structure and equipment also have a direct relationship with management; shipping companies have a weak awareness of safety in production, weak safety system construction, and unreasonable cargo stowage; for long-term ship operations, it is difficult for companies to manage It is difficult to fully implement the safety management system for all ships. Insufficient linkages and coordination mechanisms between management departments have also hindered the indepth development of coastal water traffic safety management

With the help of the model built by Zhang Hui ${ }^{[12]}$ et al. the marine ship safety system is divided into four aspects for research, and the parts are independent and mutually coupled. The evaluation result is obtained by the whole part, and each part needs to be studied separately because of different evaluation indexes.

Tab 1. Ship navigation safety assessment indicators

\begin{tabular}{ccc}
\hline First-level Indicators & First-leve2 Indicators & First-leve3 Indicators \\
\hline$A$ Environmental factors & $A_{1}$ Marine meteorological environment & $A_{11}$ sea breeze \\
& $A_{12}$ Ocean waves & $B_{11}$ Management company \\
& $B_{1}$ Ship management information & $B_{12}$ Accident history \\
$B$ Ship elements & $B_{21}$ Class \\
& $B_{2}$ Ship registration information & $B_{22}$ Boat age \\
& $B_{23}$ Ship type \\
& $B_{3}$ Ship Structure Information & $B_{24}$ Ship tonnage \\
\end{tabular}


$B_{33}$ Anti-settling

$B_{41}$ Communication and naviga-

tion equipment

$B_{4}$ Ship equipment condition

$C_{1}$ Cargo basic information

C Goods element

$C_{2}$ Cargo basic information

$D_{1}$ Historical situation

$D$ Crew elements
$B_{42}$ Safety and emergency equipment

$B_{43}$ Operating equipment

$B_{44}$ Ship power plant $C_{11}$ Cargo type

$C_{12}$ The quantity of goods

$C_{21}$ Reasonable stowage

$C_{22}$ Lashing and padding

$D_{11}$ Historical mistakes

$D_{12}$ Captain Evaluation

$D_{21}$ post

$D_{22}$ Experience at the same post
2. Navigation risk neural network algorithm design

BP neural network is a kind of multilayer feedforward neural network, which consists of input layer, hidden layer and output layer. As shown in Figure 2, it is a structure diagram of a typical three-layer BP neural network. The layers are fully interconnected. There is no interconnection between the same layers. The hidden layer can have one or more layers. The algorithm includes two aspects: the forward propagation of the signal and the backward propagation of the error. That is, the actual output is calculated in the direction from input to output, and the correction of weights and thresholds is performed in the direction from output to input.

Where, $x_{i}$ represents the input of the $j$ the input layer, $j=1,2, \cdots, M ; w_{i, j}$ represents the weight between the $i$ node of the hidden layer and the $j$ node of the input layer; $\theta_{i}$ represents the threshold of the $i$ node of the hidden layer; $\phi$ represents the excitation function of the hidden layer; $w_{k, i}$ represents the weight between the $k$ node in the output layer and the $i$ node in the hidden layer, $i=1,2, \cdots, q ; \quad a_{k}$ represents the threshold of the $k$ node of the output layer, $k=1,2, \cdots, L ; \quad o_{k}$ represents the output of the $k$ node of the output layer.

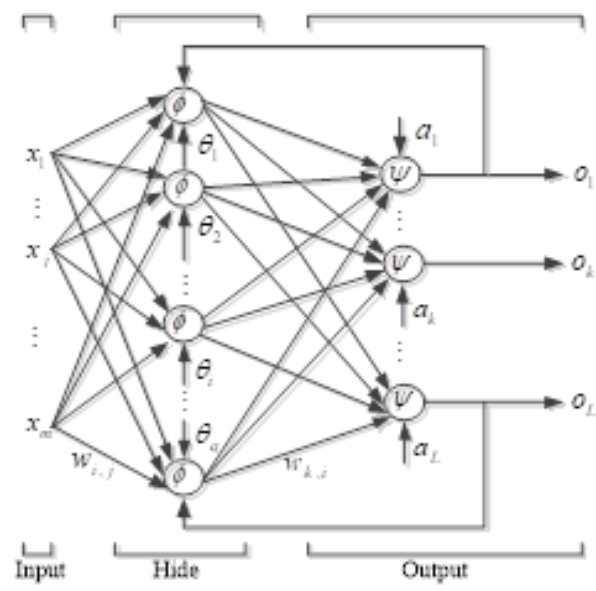

Fig 2. Three-layer BP neural network structure 


\subsection{BP neural network optimized by genetic} algorithm

Genetic algorithm to optimize BP neural network is to use genetic algorithm to optimize the initial value and threshold of BP neural network, so that the optimized BP neural network can better predict the function output. The elements of genetic algorithm to optimize BP neural network include population initialization, fitness function, selection operation, crossover operation and mutation operation. The specific network algorithm process is shown in Figure 3 below.

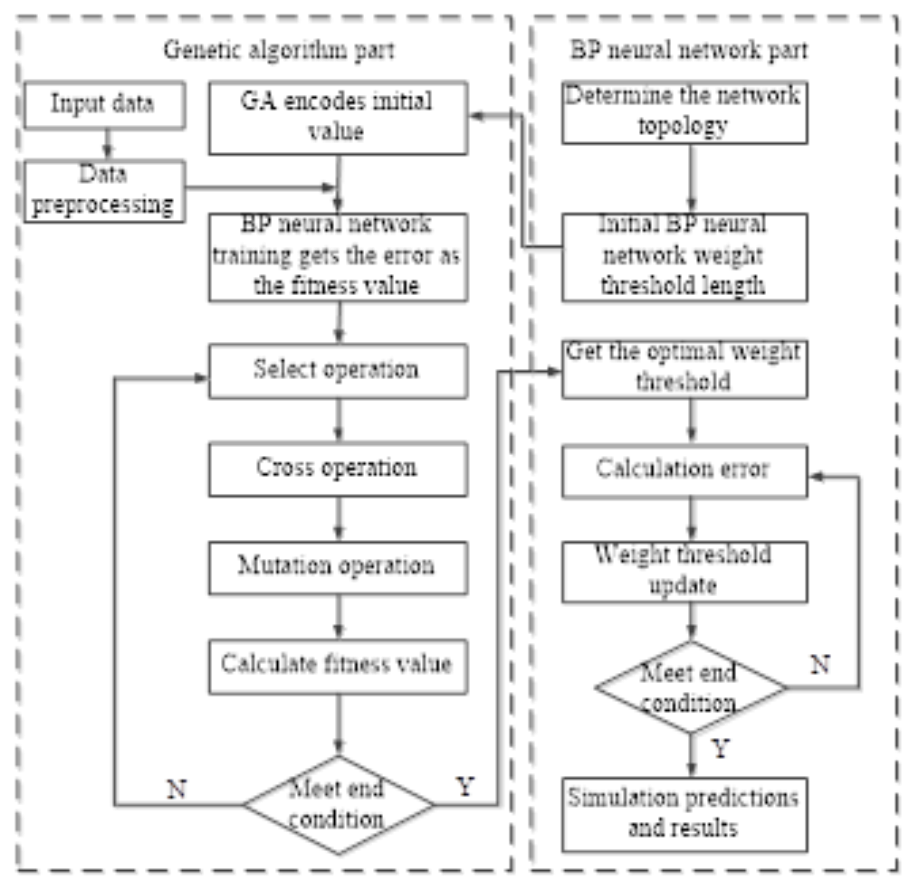

Fig 3. Genetic algorithm optimization BP neural network flow chart

\subsection{Determination of input and output layers}

\section{and neurons}

In this paper, the ship navigation safety evaluation index (see Table 1 for details) is used as the model input layer vector, and the average accident risk (evaluation of the risk value based on danger, vulnerability, and resilience) is used as the model output vector. Each index and the ship, the relationship between the risk factors is shown in Figure 4. There are 23 input layer nodes and 1 output node in the system. The number of neurons in the system will be directly

$$
\begin{aligned}
& b=\sqrt{m+n}+a \\
& b=2 n+1
\end{aligned}
$$

Where, $b$ represents the number of hidden layer neuron; $m$ represents the number of nodes in the output layer vector; $n$ denotes the number of vectors of the input layer nodes; $a$ related to the calculation results of the entire model. At present, the calculation of the number of neurons is generally determined by a trial and error method. According to the empirical formula, the possibility of the existence of the number of neurons in the hidden layer is first calculated, and a certain range of small adjustments can be made. Experiments and comparisons to determine the number of hidden layer neurons in the optimal state. The corresponding empirical formula is as follows:

represents a 1 to 10 arbitrary constant.

On this basis, the neural network can be trained after slight adjustments to the number of neurons 


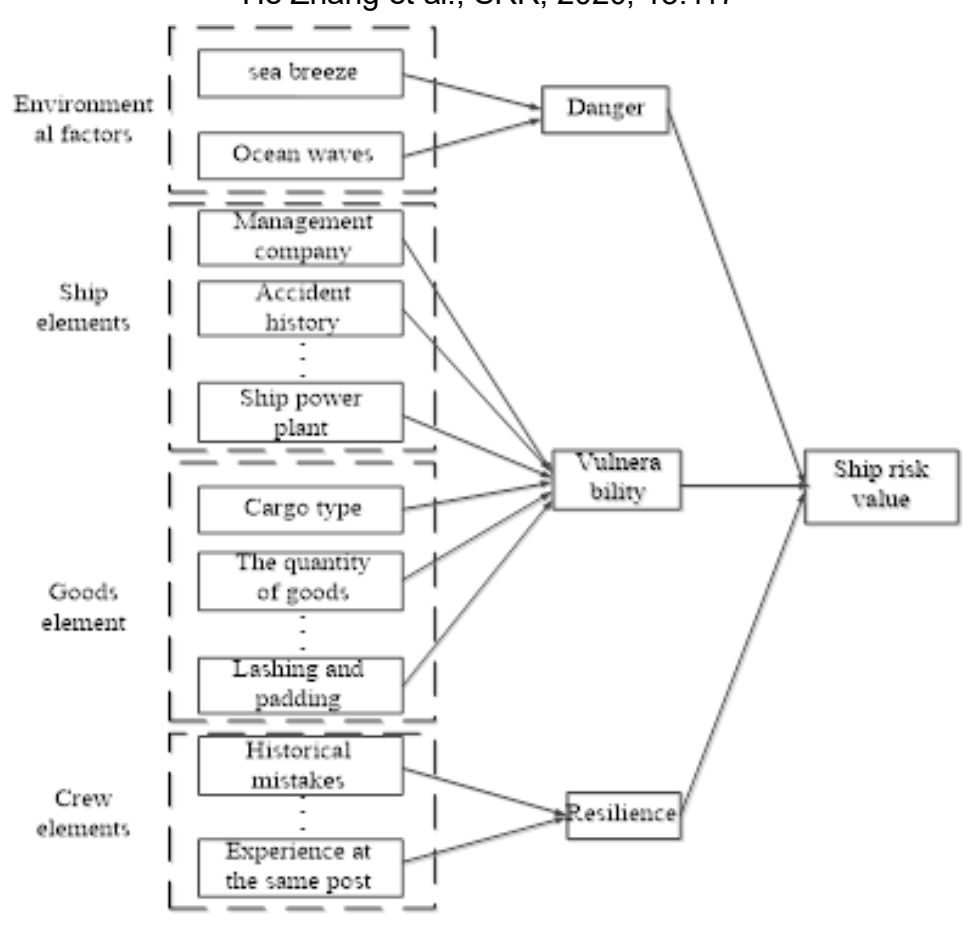

Fig 4. Diagram of indicators and ship risk factors

\section{Instance verification}

Sample data reference ${ }^{[12]}$ for statistical analysis of Clarkson ship database, marine accident report, accident database and other resources, using some data to train neural networks optimized by genetic algorithms in MATLAB environment, and finally select another set of data to verify the trained network.

As can be seen from Figure 5, the neural network is two hidden layers. The traindm algorithm is used. The total number of iterations is 10,000 .
After 45 iterations to complete the training, the total time is $1 \mathrm{~s}$. The less the number of trainings and the shorter the time, the better the network). At the end of training, the error gradient is 0.0455 , which is much larger than the training default of 10-5, which means that the network error at this time still shows a rapid downward trend. Figure 6 is a graph of the training error descent gradient. It can be seen from the figure that the network converges continuously during the learning process.

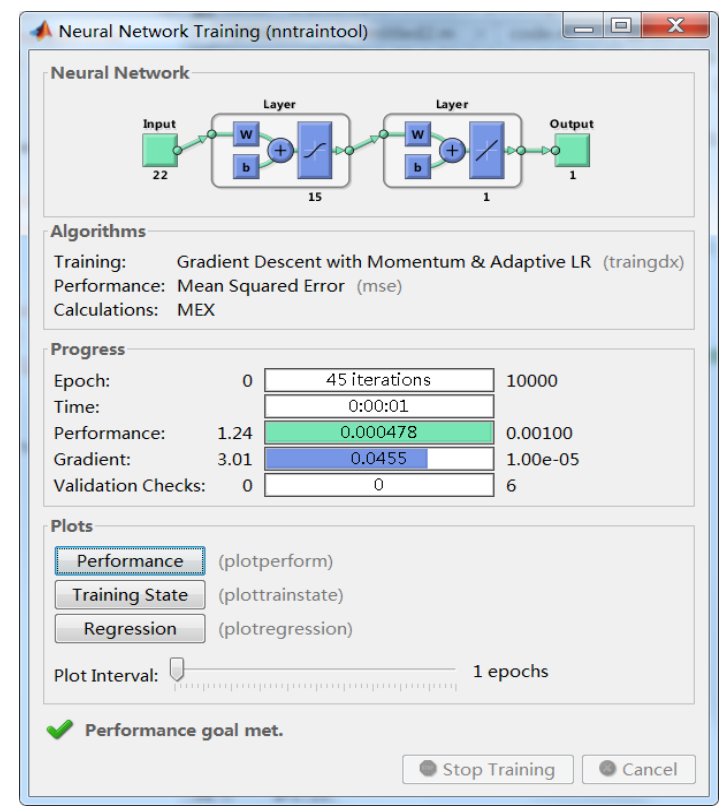

Fig.5 BP neural network training results 


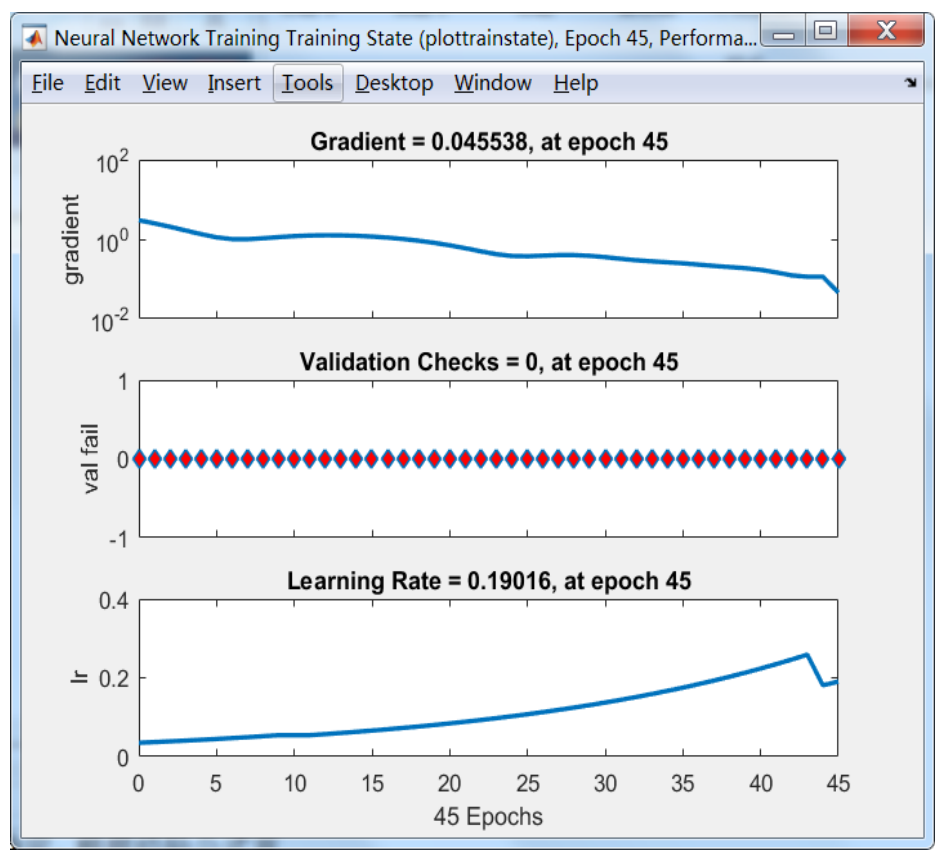

Fig.6 Training error descent gradient

Figures 7 and 8 respectively show the compari- degree of fitting is high, and the error between son of the network training results and actual re- the test result and the actual result is relatively sults for the ship risk value, and the difference small. At the same time, it also shows that the between the training results and the actual re- optimized BP neural network can evaluate the sults. From the figure, it can be seen that the op- risk value of the ship in a certain sense when the timized BP neural network has a value for the error is allowed.

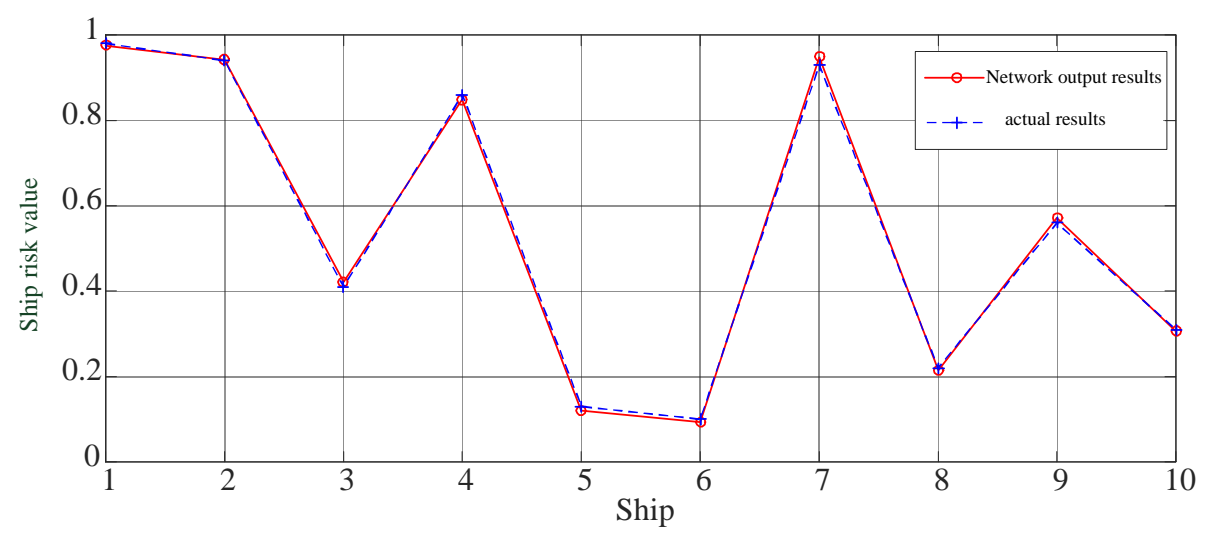

Fig.7 Comparison of ship risk value fitting curves

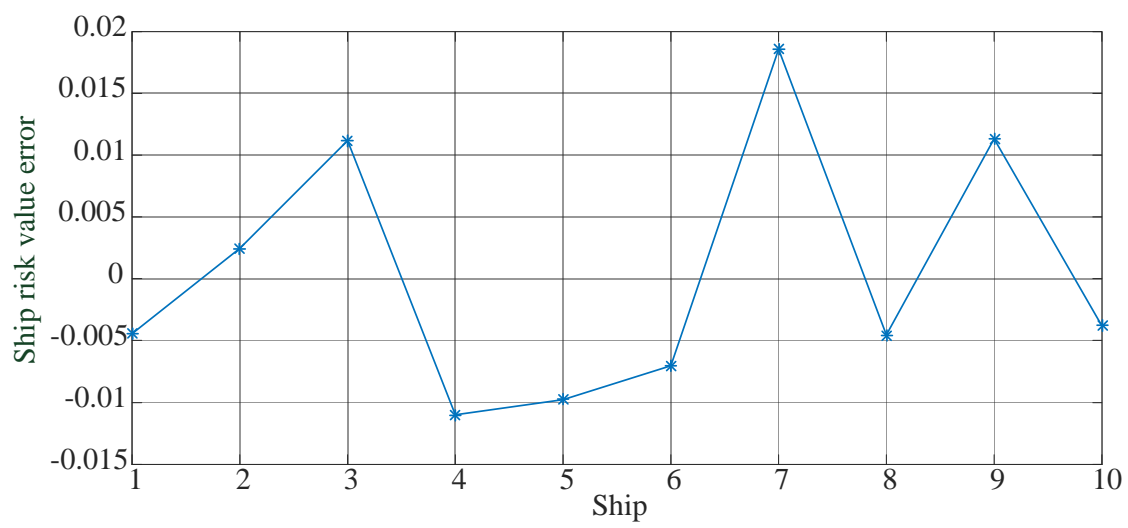

Fig.8 Ship risk value error curve 


\section{Conclusion}

Combining various influencing factors, a ship navigation safety evaluation system is constructed. The principle of BP neural network algorithm is used to perform self-learning training on the input sample data. In order to make the learning results more efficient and accurate, a genetic algorithm is introduced to find the optimal fitness value corresponding to the individual, and the weight and threshold of the BP neural network are optimized online. Through practical calculations, it is clear that the BP neural network optimized by genetic algorithm can eliminate the construction of complex influencing factor relationships in ship navigation safety assessment, autonomous learning and training, and the risk assessment of ships is feasible and effective to a certain extent.

\section{References}

[1] Xie Yiyang , Liu Dagang, Wu Danzhu , et al. Risk Prediction Technology of Ship Safety in the Yellow Sea and Bohai Sea during Severe Weathers[J]. Journal of Catastrophology, 2014, 29(1): 136-143.

[2] Mengxuan Wang. Li Wei. Safe Assessment of Containerships Based on ReliefF-ANFIS[J]. Ship Electronic Engineering, 2018, 38(07): 100$105+121$.

[3] Duan Aiyuan, Zhao Yao, Preliminary evaluation on ship safety based on unascertained measure[J]. Journal of Shanghai Maritime University, 2007(02): 21-23+27.

[4] Wang Zhijuan, Wei Hongchang . Precise Ship Attitude Prediction Technology based on Improved Neural Network[J]. Ship Science and Technology, 2019,41(12): 37-39.

[5] Xiao Jingkun, Yin Peihai , Lin Jianguo et al. Identification of ship's oil spill risk degree of sea area by using BP artificial network[J]. Marine Environmental Science, 2002(04): 42-45.

[6] Chen Xiaoting, Wu Jingna, Lu Haixia et al. Analysis and evaluation of nutritional components in the muscle of decapterus maruadsi[J]. Fishery Modernization, 2016,43(01):47-51+61.

[7] Chandre G C , Mayya S G . Comparison of Back Propagation Neural Network and Genetic Algorithm Neural Network for Stream Flow Prediction[J]. Journal of Computational Environmental Sciences, 2014, 2014:1-6.

[8] Zhang Xinfang, Guan Keping, Research on Influencing Factors and Safety Evaluation of Ship Navigation Safety[J]. China Water Transport, 2015, 15(11):50-52.

[9] Wu Haihua. Analysis of factors affecting ship navigation safety[J]. China Water Transport, 2006(5).

[10] Liang Kailin . Analysis on human factors in maritime traffic accident based on CREAM[D]. Dalian Maritime University, 2014.

[11] Zhang hui. The ship Capsizing Risk Assessment based on BP Neural Network[D]. Dalian Maritime University, 2018.

[12] Zhang hui. The ship Capsizing Risk Assessment based on BP Neural Network[D]. Dalian Maritime University, 2018. 